Discrete Comput Geom 30:437-452 (2003)

DOI: $10.1007 / \mathrm{s} 00454-003-2824-\mathrm{x}$

\title{
Complexity of the Delaunay Triangulation of Points on Polyhedral Surfaces
}

\author{
Dominique Attali ${ }^{1}$ and Jean-Daniel Boissonnat ${ }^{2}$ \\ ${ }^{1}$ LIS, ENSIEG, Domaine Universitaire, \\ BP 46, 38402 Saint Martin d'Hères Cedex, France \\ Dominique.Attali@inpg.fr \\ ${ }^{2}$ INRIA, Unité de Recherche Sophia Antipolis, \\ 2004 Route des Lucioles, BP 93, 06904 Sophia-Antipolis, France \\ Jean-Daniel.Boissonnat@sophia.inria.fr
}

\begin{abstract}
It is well known that the complexity of the Delaunay triangulation of $n$ points in $\mathbb{R}^{d}$, i.e., the number of its simplices, can be $\Omega\left(n^{\lceil d / 2\rceil}\right)$. In particular, in $\mathbb{R}^{3}$, the number of tetrahedra can be quadratic. Put another way, if the points are uniformly distributed in a cube or a ball, the expected complexity of the Delaunay triangulation is only linear. The case of points distributed on a surface is of great practical importance in reverse engineering since most surface reconstruction algorithms first construct the Delaunay triangulation of a set of points measured on a surface.

In this paper we bound the complexity of the Delaunay triangulation of points distributed on the boundary of a given polyhedron. Under a mild uniform sampling condition, we provide deterministic asymptotic bounds on the complexity of the three-dimensional Delaunay triangulation of the points when the sampling density increases. More precisely, we show that the complexity is $O\left(n^{1.8}\right)$ for general polyhedral surfaces and $O(n \sqrt{n})$ for convex polyhedral surfaces.

Our proof uses a geometric result of independent interest that states that the medial axis of a surface is well approximated by a subset of the Voronoi vertices of the sample points.
\end{abstract}

\section{Introduction}

It is well known that the complexity of the Delaunay triangulation of $n$ points in $\mathbb{R}^{d}$, i.e., the number of its simplices, can be $\Omega\left(n^{\lceil d / 2\rceil}\right)$. In particular, in $\mathbb{R}^{3}$, the number of tetrahedra can be quadratic. Put another way, if the points are uniformly distributed in a cube or a ball, the expected complexity of the Delaunay triangulation is only linear [8], [9]. 


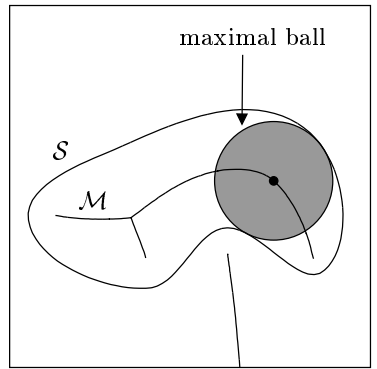

(a)

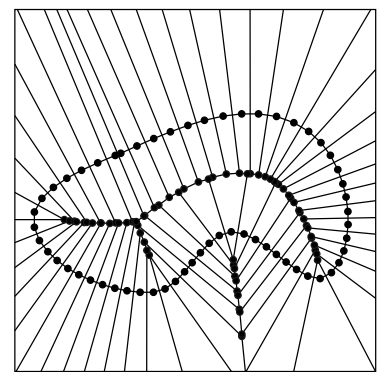

(b)

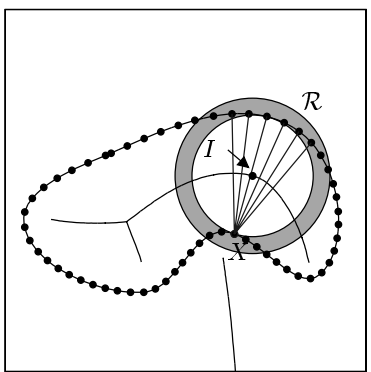

(c)

Fig. 1. Overview of the proof in $\mathbb{R}^{2}$. (a) A curve $\mathcal{S}$ and its medial axis $\mathcal{M}$. (b) Voronoi graph of sample points. (c) The inner Delaunay neighbors of $X$ that are far away from $X$ lie in a small region $\mathcal{R}$.

The case of points distributed on a surface is of great practical importance in reverse engineering since most surface reconstruction algorithms first construct the Delaunay triangulation of a set of points measured on a surface, see, e.g., [1] and [5]. The time complexity of those methods therefore crucially depends on the complexity of the triangulation of points scattered over a surface in $\mathbb{R}^{3}$. Moreover, since output-sensitive algorithms are known for computing Delaunay triangulations [7], better bounds on the complexity of the Delaunay triangulation would immediately imply improved bounds on the time complexity of computing the Delaunay triangulation.

A first result has been recently obtained by Golin and $\mathrm{Na}$ [11]. They proved that the expected complexity of three-dimensional Delaunay triangulations of random points on convex polytopes is $\Theta(n)$. The case of points on a cylinder has been considered by Erickson who proved that, even if the cylinder is well-sampled, the complexity of the Delaunay triangulation may be $\Omega(n \sqrt{n})$ [10]. Erickson's paper also contains lower bounds for contrived surfaces with a nonbounded ratio between the diameter and the minimum local feature size, a case we exclude here.

In this paper we consider the case of points distributed on the boundary of a given polyhedron. Under a mild uniform sampling condition, we provide deterministic asymptotic bounds on the complexity of the three-dimensional Delaunay triangulation of the points when the sampling density increases. More precisely, we show that the complexity is $O\left(n^{1.8}\right)$ for general polyhedral surfaces and $O(n \sqrt{n})$ for convex polyhedral surfaces. The intuition behind our result is the following. When a surface $\mathcal{S}$ is well-sampled, the circumcenters of the Delaunay simplices with a long edge are close to the medial axis $\mathcal{M}$ of $\mathcal{S}$ (see Fig. 1(b)). It follows that the Delaunay neighbors of a point $X$ that are sufficiently far away from $X$ lie in a small region $\mathcal{R}$ between two spheres centered at a point $I$ of $\mathcal{M}$ (see Fig. 1(c)). In the case of a polyhedral surface, the intersection of $\mathcal{R}$ and $\mathcal{S}$ is contained in a bounded number of small disks and therefore $X$ can only have a small number of Delaunay neighbors.

\section{Medial Axis Approximation}

Our combinatorial bound on the complexity of the Delaunay triangulation of a light uniform sample $\mathcal{A}$ is based on a geometric result (Theorem 10 below) that states that the 
medial axis of the surface is well approximated by a subset of the Voronoi vertices of $\mathcal{A}$. Before stating and proving this result, we recall the definition of the medial axis of a surface and define uniform samples. In this section we do not impose that the surface be polyhedral.

\subsection{Definitions}

The medial axis was first introduced by Blum in the field of image analysis as a tool for shape description. Since then the medial axis has been intensively studied. In this section we recall definitions and properties related to the medial axis. Other results on the medial axis can be found in [6], [12], [14], [13], and [3].

The medial axis of a subset $\mathcal{F}$ of $\mathbb{R}^{3}$ can be defined using the concept of a maximal ball.

Definition 1 (Maximal Ball). Let $\mathcal{F} \subset \mathbb{R}^{3}$. An open ball $B$ is said to be maximal in $\mathcal{F}$ if and only if for any open ball $B^{\prime}, B \subseteq B^{\prime} \subseteq \mathcal{F} \Rightarrow B=B^{\prime}$.

Definition 2 (Medial Axis of an Object). Let $\mathcal{F} \subset \mathbb{R}^{3}$. The medial axis of $\mathcal{F}$ is the closure of the centers of the maximal balls of $\mathcal{F}$.

This definition can be extended to surfaces as follows:

Definition 3 (Medial Axis of a Surface). $\quad$ Let $\mathcal{S}$ be an embedded 2-manifold. We call the medial axis of $\mathcal{S}$ the medial axis of $\mathbb{R}^{3} \backslash \mathcal{S}$.

At any point $X \in \mathcal{S}$, we associate the local feature size. The concept of local feature size was first introduced in the context of surface reconstruction by Amenta and Bern [1].

Definition 4 (Local Feature Size). The local feature size lfs $(X)$ at a point $X \in \mathcal{S}$ is the distance from $X$ to the medial axis of $\mathcal{S}$.

In the most general case there can be an infinite number of maximal balls through a given point $X \in \mathcal{S}$. Let $R$ be the radius of any maximal ball through $X$ :

$$
\operatorname{lfs}(X) \leq R
$$

However, if the normal to $\mathcal{S}$ at $X$ is defined, there are exactly two maximal balls through $X$, one on each side of the tangent plane to $\mathcal{S}$ at $X$.

We distinguish two types of points on $\mathcal{S}$, singular and regular points:

Definition 5 (Singular and Regular Points). A point $X \in \mathcal{S}$ is said to be regular iff (1) the normal to $\mathcal{S}$ at $X$ is defined, (2) the two maximal balls through $X$ touch $\mathcal{S}$ in at least two distinct points $X$ and $Y \neq X$. A point is said to be singular iff it is not regular. 
For polyhedral surfaces, the two conditions are equivalent. The singular points are the points of the edges of the polyhedral surface. For smooth surfaces, the normal is defined everywhere and the singular points are the points of the ridges of the surface [3].

\subsection{Uniform Samples}

Our result holds on a sampling condition that roughly says that the sample should be uniform but not arbitrarily dense locally. This is made precise through the following definitions.

Definition 6 (Uniform $\varepsilon$-Sample). A set of points $\mathcal{A} \in \mathcal{S}$ is called a uniform $\varepsilon$-sample of $\mathcal{S}$ iff for every point $X \in \mathcal{S}$, the ball $B(X, \varepsilon)$ contains at least one point of $\mathcal{A}$.

Definition 7 (Light Uniform $\varepsilon$-Sample). A uniform $\varepsilon$-sample is said to be light iff for every point $X \in \mathcal{S}$, the ball $B(X, r)$ contains $O\left(r^{2} / \varepsilon^{2}\right)$ points of $\mathcal{A}$.

Observe that our definition of a light uniform $\varepsilon$-sample does not impose any lower bound on the minimal distance between two sample points.

Amenta and Bern have introduced a different definition of an $\varepsilon$-sample [1]. The originality of their definition is to enforce the sample to fit the surface shape locally. According to their definition, point density is high where the surface has high curvature or where the object or its complement is thin. However, if the local feature size vanishes, an $\varepsilon$-sample, as defined in [1], will have an infinite number of points, which is not satisfactory for our purpose.

Erickson has introduced a notion of a uniform sample that is related to our notion of a light uniform sample but forbids points to be too close (which our definition allows) [10].

In the rest of the paper, $\mathcal{A}$ denotes a light uniform $\varepsilon$-sample and $\mathcal{S}$ a compact fixed surface. In particular, we assume that quantities like the area or the diameter of $\mathcal{S}$ are fixed and do not depend on $\varepsilon$. Hence the number of points $n$ of $\mathcal{A}$ is bounded and $n=O\left(1 / \varepsilon^{2}\right)$. We provide asymptotic results when the sampling density increases, i.e., when $\varepsilon$ tends to zero.

\subsection{Medial Axis Approximation}

The goal of this section is to prove that the circumcenters of the Delaunay tetrahedra with long edges converge toward the medial axis (Theorem 10). Our result improves on related results obtained by Amenta and Kolluri [2] and Boissonnat and Cazals [4]. In [2] the convergence is established for a subset of the Voronoi vertices called the poles. The poles of a sample point $X$ are the two vertices of its Voronoi cell farthest from $X$, one on either side of the surface. In this paper we establish a result for a larger class of Voronoi vertices. We also allow the normal to the surface not to be defined everywhere in order to be able to apply this result to polyhedral surfaces later. 
In this section, as well as in the rest of the paper, the notation $f \ll g$ (or $f=o(g)$ ) means that $f / g$ tends to zero when $\varepsilon$ tends to zero. The notation $f \approx g$ means that $f-g \ll g$. The notation $f \lesssim g$ means that $f \leq g$ or $f \approx g$.

We start with a technical lemma. In this lemma we consider four points $X, A, I$, and $V$ and establish a bound on $\|V I\|$ assuming that $\|X A\|$ is large enough. $X$ and $A$ will later designate two sample points on the surface that are adjacent in the Delaunay triangulation. $I$ will designate the center of one of the two maximal balls through $X . V$ will designate a vertex of the Voronoi facet dual to the Delaunay edge $[X A]$.

Lemma 8. Let $\rho$ and $\theta$ be two positive functions of $\varepsilon$ such that $\rho \ll 1$ and $\theta \ll 1$. Let $A, X, I$, and $V$ be four points satisfying the following conditions:

1. $\|V A\|=\|V X\|$,

2. $\|X I\|=R$,

3. $\|A I\|=R(1+\rho)$,

4. $\angle(\overrightarrow{X I}, \overrightarrow{X V})=\theta$,

5. $\|X A\|=2 l$.

If $\rho R^{2} / l^{2} \ll 1$ and $R \theta / l \ll 1$, then we have

$$
\|V I\| \lesssim \frac{\theta R^{2}}{l}+\frac{R^{3} \rho}{2 l^{2}}
$$

Proof. Refer to Fig. 2. Assume the three points $A, X$, and $I$ are given. Let $\mathcal{H}$ be the plane that bisects the points $X$ and $A$. Let $\mathcal{C}$ be the circular cone generated by the lines

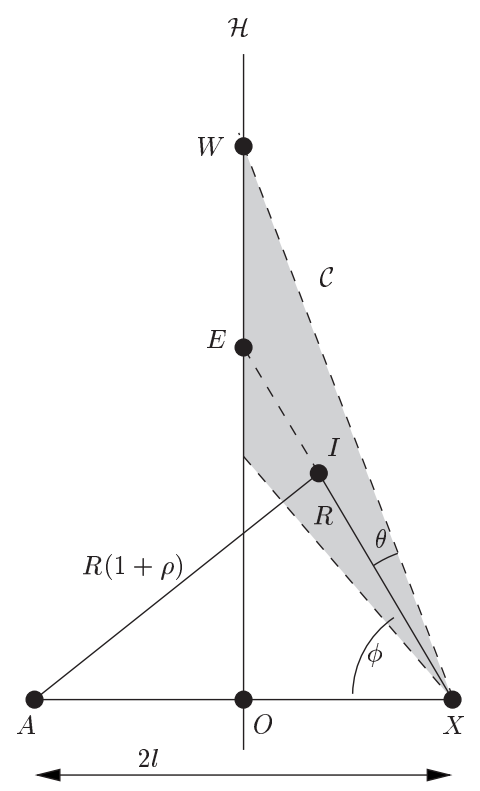

Fig. 2. For the proof of Lemma 8. 
passing through $X$ and forming an angle $\theta$ with the line $(X I)$. Point $V$ lies in plane $\mathcal{H}$ and on cone $\mathcal{C}$. Therefore, $V$ lies on the ellipse $\mathcal{H} \cap \mathcal{C}$. Let $W$ be the point on this ellipse which is the farthest from point $I$. In addition, let $O$ be the midpoint of segment $[A X]$ and let $E$ be the intersection point of line $(X I)$ with $\mathcal{H}$. Let us bound $\|W I\|$. By the triangle inequality,

$$
\|W I\| \leq\|W E\|+\|E I\| .
$$

Let $\varphi$ be the angle between the two vectors $\overrightarrow{X O}$ and $\overrightarrow{X I}$. Since the lengths of the three sides of triangle $(A X I)$ are known, we have

$$
\begin{aligned}
\cos \varphi & =\frac{-\rho(2+\rho) R^{2}+4 l^{2}}{4 l R} \\
& \approx \frac{l}{R} .
\end{aligned}
$$

Using the expression of $\cos \varphi$, we can bound $\|W E\|$ :

$$
\begin{aligned}
\|W E\| & =l(\tan (\varphi+\theta)-\tan \varphi) \\
& =\frac{l \sin \theta}{\cos (\varphi+\theta) \cos \varphi} \\
& \approx \frac{\theta R^{2}}{l} .
\end{aligned}
$$

Let us now bound $\|E I\|$ :

$$
\begin{aligned}
\|E I\| & =\frac{l}{\cos \varphi}-R \\
& =\frac{4 l^{2} R}{4 l^{2}-\rho(2+\rho) R^{2}}-R \\
& \approx \frac{R^{3} \rho}{2 l^{2}} .
\end{aligned}
$$

Finally, we get

$$
\|W I\| \lesssim \frac{\theta R^{2}}{l}+\frac{R^{3} \rho}{2 l^{2}}
$$

Let $V$ denote a vertex of the Voronoi cell of $X$ in the Voronoi diagram of $\mathcal{A}$. Let $I$ be the center of one of the two maximal balls through $X$. By slightly adapting a result of Amenta and Bern [1, Lemma 5], we can bound $\theta=\angle(\overrightarrow{X I}, \overrightarrow{X V})$.

Lemma 9. Let $X$ be a regular point of $\mathcal{S}$. Assume $\|V X\| \geq \varepsilon$ and $\operatorname{lfs}(X) \geq \varepsilon$. Then the angle at $X$ between the normal to $\mathcal{S}$ at $X$ and the vector to $V$ (oriented so that the angle is acute) is at most $\arcsin (\varepsilon /\|V X\|)+\arcsin (\varepsilon / \mathrm{lfs}(X))$.

The next proposition states that the circumcenters of the Delaunay tetrahedra with long edges converge toward the medial axis when $\varepsilon$ tends to zero. 
Theorem 10 (Medial Axis Approximation). Let $\mathcal{A}$ be a uniform $\varepsilon$-sample of $\mathcal{S}$ and let $X \in \mathcal{A}$ be a regular point of $\mathcal{S}$. Let $[X A]$ be a Delaunay edge and let $V$ a vertex in the Voronoi facet dual to the Delaunay edge $[X A]$. Let $B(I, R)$ be the one maximal ball through $X$ such that $\overrightarrow{X V} \cdot \overrightarrow{X I}>0$. Let $Y$ be a point of $\partial B(I, R) \cap \mathcal{S}$ distinct from $X$. Assume that:

1. $\varepsilon \ll \operatorname{lfs}(X)$,

2. $\|X Y\|^{2} / 4 R^{2} \gg \varepsilon / \mathrm{lfs}(X)$,

3. $\|X A\|^{2} / 4 R^{2} \gg \varepsilon / \operatorname{lfs}(X)$.

Then

$$
\|V I\| \lesssim R \frac{\varepsilon}{\operatorname{lfs}(X)} \max \left(\frac{10 R^{2}}{\|X Y\|^{2}},\left(1+\frac{2 R}{\|X A\|}\right)^{2}\right)
$$

Proof. Since $\|V X\| \geq \frac{1}{2}\|X A\|$ and $R \geq \operatorname{lfs}(X)$ and using assumptions 1 and 3 , we have

$$
\frac{\varepsilon}{\|V X\|} \leq \frac{2 \varepsilon}{\|X A\|} \ll \frac{\varepsilon}{R} \sqrt{\frac{\operatorname{lfs}(X)}{\varepsilon}} \leq \sqrt{\frac{\varepsilon}{\operatorname{lfs}(X)}} \ll 1
$$

Therefore, we can apply Lemma 9. The angle $\theta$ between the vectors $\overrightarrow{X I}$ and $\overrightarrow{X V}$ is at most

$$
\theta \leq \arcsin \left(\frac{\varepsilon}{\|V X\|}\right)+\arcsin \left(\frac{\varepsilon}{\operatorname{lfs}(X)}\right) \approx \frac{\varepsilon}{\|V X\|}+\frac{\varepsilon}{\operatorname{lfs}(X)} .
$$

Let $\Sigma_{v}$ and $\Sigma_{p}$ be two spheres passing through $X$. The first one, $\Sigma_{v}$, is centered at $V$ and is therefore empty. The second one, $\Sigma_{p}$, has radius $R$ and is centered on the half-line going from $X$ to $V$. We denote the center of $\Sigma_{p}$ by $P$.

Two cases must be considered. First, assume $V$ is farther from $X$ than $P$ (see Fig. 3, left). In this case the ball bounded by $\Sigma_{p}$ is contained in the ball bounded by $\Sigma_{v}$ and therefore does not contain any point of $\mathcal{A}$ in its interior. Let $A_{y}$ be one of the sample points in the neighborhood of $Y$ at distance at most $\varepsilon$ from $Y$, and let $\Sigma_{c}$ be the sphere tangent to $\Sigma_{p}$ at $X$ and passing through $A_{y}$. We denote the center of $\Sigma_{c}$ by $C$. Since $V$ lies between $C$ and $P,\|V I\| \leq\|C I\|$. We apply Lemma 8 with $A_{y}, X, I$, and $C$. The five items of Lemma 8 are fulfilled. Indeed, since $\|V X\| \geq\|P X\|=R \geq \operatorname{lfs}(X)$, (2) implies $\theta \lesssim 2 \varepsilon / \operatorname{lfs}(X)$; as noticed above, $\rho \leq \varepsilon / R \leq \varepsilon / \operatorname{lfs}(X)$; and $2 l=\left\|X A_{y}\right\| \geq\|X Y\|-\varepsilon$. We first note that $\|X Y\| \gg \varepsilon$. Indeed, using again the inequality $R \geq \operatorname{lfs}(X)$ and assumptions 2 and 1 , we have

$$
\frac{\|X Y\|}{2} \gg R \sqrt{\frac{\varepsilon}{\operatorname{lfs}(X)}} \geq \sqrt{\operatorname{lfs}(X) \varepsilon} \gg \varepsilon
$$

We now use our assumption $\|X Y\|^{2} / 4 R^{2} \gg \varepsilon / \operatorname{lfs}(X)$ to prove that $l \gg R \theta$ and $l^{2} \gg$ $\rho R^{2}$. Indeed,

$$
l \geq \frac{\|X Y\|}{2}-\varepsilon \gg R \sqrt{\frac{\varepsilon}{\operatorname{lfs}(X)}} \gg R \frac{2 \varepsilon}{\operatorname{lfs}(X)} \gtrsim R \theta
$$



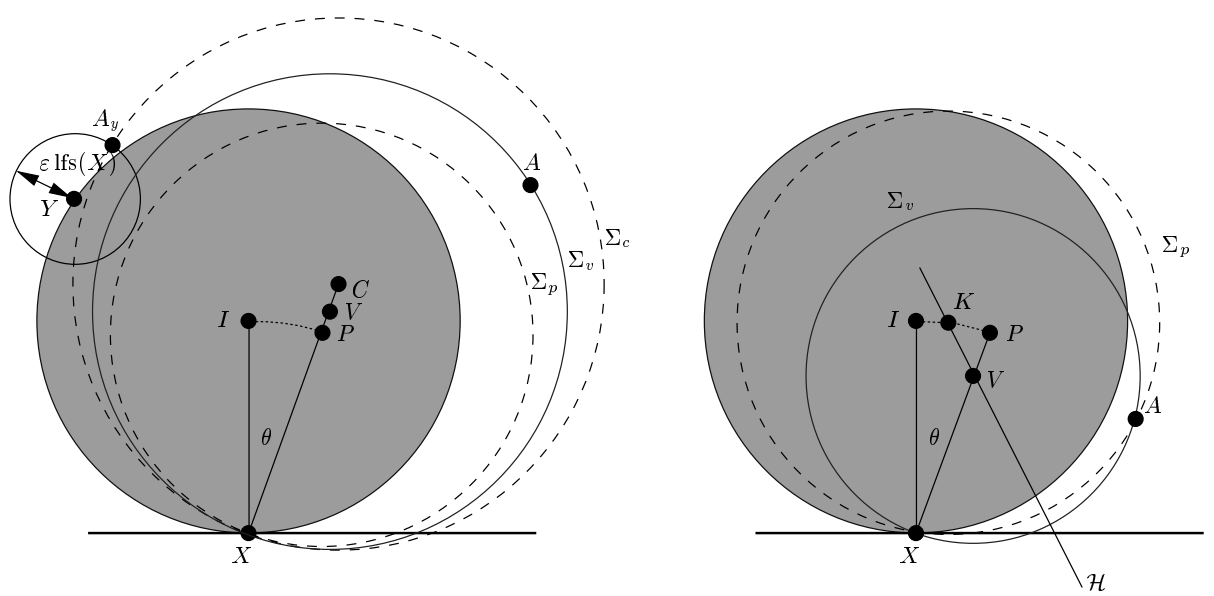

Fig. 3. The two cases for the proof of Theorem 10 .

and

$$
l^{2} \geq\left(\frac{\|X Y\|}{2}-\varepsilon\right)^{2} \gg R^{2} \frac{\varepsilon}{\operatorname{lfs}(X)} \geq R^{2} \frac{\varepsilon}{R} \geq \rho R^{2} .
$$

Since $R /\|X Y\| \geq \frac{1}{2}$, Lemma 8 then implies

$$
\|V I\| \leq\|C I\| \lesssim \frac{R^{2} \theta}{l}+\frac{R^{3} \rho}{2 l^{2}} \lesssim \frac{4 R^{2}}{\|X Y\|} \frac{\varepsilon}{\operatorname{lfs}(X)}\left(1+\frac{R}{2\|X Y\|}\right) \leq R \frac{\varepsilon}{\operatorname{lfs}(X)} \frac{10 R^{2}}{\|X Y\|^{2}} .
$$

Consider now the second case and assume that $V$ is closer to $X$ than $P$. Let $\mathcal{H}$ be the plane that bisects $X$ and $A$. Since $\mathcal{H}$ contains $V, P$ lies in the half-space limited by $\mathcal{H}$ that contains $A$. Since $I$ lies in the other half-space, the circle $\operatorname{arc} I P$ (centered at $X$ and of radius $R$ ) must intersect $\mathcal{H}$ at some point $K$. We now apply Lemma 8 to $A, X, K$, and $V$. Notice that $\rho=0$ and $\theta^{\prime}=\angle(\overrightarrow{X K}, \overrightarrow{X V})$. Let $l=\frac{1}{2}\|X A\|$. Since $\rho=0, l^{2} \gg \rho R^{2}$. By inequality (1), $\varepsilon /\|V X\| \ll \sqrt{\varepsilon / \operatorname{lfs}(X)}$ and thus

$$
\theta^{\prime} \leq \theta \lesssim \frac{\varepsilon}{\|V X\|}+\frac{\varepsilon}{\operatorname{lfs}(X)} \ll \sqrt{\frac{\varepsilon}{\operatorname{lfs}(X)}}+\frac{\varepsilon}{\operatorname{lfs}(X)} \approx \sqrt{\frac{\varepsilon}{\operatorname{lfs}(X)}} .
$$

We now use our assumption $\|X A\|^{2} / 4 R^{2} \gg \varepsilon / \operatorname{lfs}(X)$ to prove that $l \gg R \theta^{\prime}$ :

$$
l=\frac{1}{2}\|X A\| \gg R \sqrt{\frac{\varepsilon}{\operatorname{lfs}(X)}} \gg R \theta^{\prime} .
$$

Lemma 8 then gives

$$
\|V K\| \lesssim \frac{R^{2} \theta^{\prime}}{l}
$$


Therefore

$$
\begin{aligned}
\|V I\| & \leq\|V K\|+\|K I\| \\
& \lesssim \frac{R^{2} \theta}{l}+R \theta \\
& \lesssim R \varepsilon\left(\frac{1}{\|V X\|}+\frac{1}{\operatorname{lfs}(X)}\right)\left(1+\frac{2 R}{\|X A\|}\right) \\
& \lesssim R \frac{\varepsilon}{\operatorname{lfs}(X)}\left(1+\frac{2 R}{\|X A\|}\right)^{2} .
\end{aligned}
$$

Note that the proposition above makes no assumption on the contact point $Y$ of the maximal ball through $X$. $Y$ may or may not be regular. It does not matter either that the local feature size vanishes at $Y$.

We can also note that the result depends on the ratio $\varepsilon / \operatorname{lfs}(X)$. In fact, if $r=\varepsilon / \operatorname{lfs}(X)$, we get an equivalent approximation theorem for $r$-samples, as defined by Amenta and Bern in [1].

\section{Polyhedral Surfaces}

\subsection{Definition and Properties}

We call the boundary of a bounded polyhedron with a finite number of faces the polyhedral surface. The medial axis of a polyhedral surface is composed of pieces of planes and quadrics (see Fig. 6(b)). In this section we establish four properties concerning polyhedral surfaces that will be used in the next section in order to bound the complexity of the Delaunay triangulation of points distributed on a polyhedral surface.

Property 11. Let $\mathcal{S}$ be a polyhedral surface. For every facet $\mathcal{F} \subset \mathcal{S}$ and every point $X \in \mathcal{F}, B(X$, lfs $(X)) \cap \mathcal{S}$ is a disk contained in $\mathcal{F}$.

Proof. Refer to Fig. 4. Let $\mathcal{S}_{f}=\mathcal{S} \backslash \mathcal{F}$ be the facets of $\mathcal{S}$ different from $\mathcal{F}$. To get a contradiction, we assume that $B(X$, lfs $(X)) \cap \mathcal{S}_{f} \neq B(X$, lfs $(X)) \cap \mathcal{F}$. We consider the point $Y$ of $\mathcal{S}_{f}$ closest to $X$. $Y$ belongs to a facet $\mathcal{F}^{\prime} \neq \mathcal{F}$.

If $Y$ is on an edge of $\mathcal{F}$ (see Fig. 4(a)), $\|X Y\|=1$ ffs $(X)$ since the edges of $\mathcal{S}$ are included in the medial axis of $\mathcal{S}$. Hence the intersection of $B(X$, lfs $(X))$ with the support plane of $\mathcal{F}$ is a disk $\mathcal{D} \subset \mathcal{F}$. Moreover, since $B(X, \operatorname{lfs}(X)) \cap \mathcal{S}$ is a topological disk [4, Proposition 14], $B(X$, lfs $(X)) \cap \mathcal{S}=\mathcal{D}$.

If $Y$ does not belong to $\mathcal{F}$ (see Fig. 4(b)). In that case, $B(X$, lfs $(X)) \cap \mathcal{S}$ consists of at least two connected components, one included in $\mathcal{F}$ and the other containing $Y$. This contradicts the fact that $B(X, \operatorname{lfs}(X)) \cap \mathcal{S}$ is a topological disk.

Property 12. Let $\mathcal{S}$ be a polyhedral surface and let $X$ be a regular point of $\mathcal{S}$. Let $B$ a maximal ball centered on the medial axis of $\mathcal{S}$ that passes through $X$ and $Y$. Then $\operatorname{lfs}(X) \leq\|X Y\|$. 


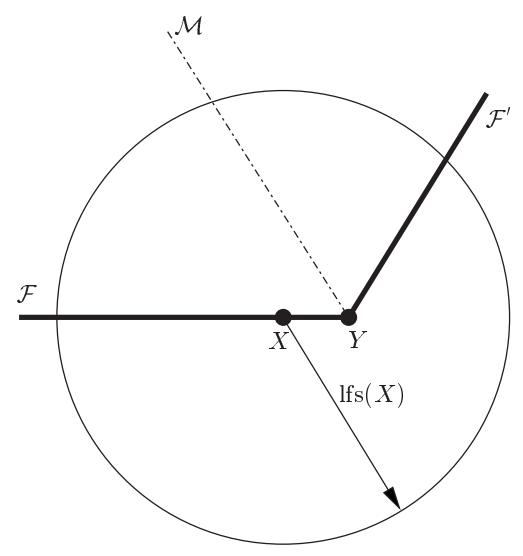

(a)

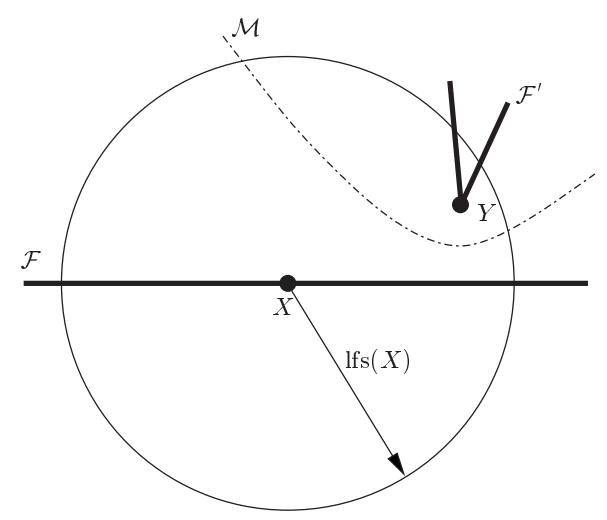

(b)

Fig. 4. For the proof of Property 11.

Proof. The proof is by contradiction. We assume $\|X Y\|<\operatorname{lfs}(X)$. Since $X$ is a regular point, $X$ and $Y$ do not belong to the same facet. Let $\mathcal{F}$ be the facet containing $X$. $Y$ belongs to $B(X, \operatorname{lfs}(X)) \cap \mathcal{S}$ and $Y$ does not belong to $\mathcal{F}$, which contradicts Proposition 11 .

Property 13. Let $\mathcal{P}$ be a convex polyhedron and let $\mathcal{S}$ be the boundary of $\mathcal{P}$. Let $R$ be the radius of the maximal ball (included in $\mathcal{P}$ ) that passes through $X \in \mathcal{S}$. For every regular point $X \in \mathcal{S}$, we have

$$
1 \leq \frac{R}{\operatorname{lfs}(X)} \leq \frac{1}{\cos \left(\alpha_{\max } / 2\right)}
$$

where $\alpha_{\max } \in[0, \pi[$ designates the greatest angle between any two faces of $\mathcal{P}$.

Proof. Refer to Fig. 5. We have already noticed that $1 \leq \mathrm{lfs}(X) / R$. Let $\mathcal{F}$ be the facet of $\mathcal{S}$ containing $X$. Let $\operatorname{Terr}(\mathcal{F})=\{Y \in \mathcal{P}, d(Y, \mathcal{F})=d(Y, \partial \mathcal{P}\}$. Since $\mathcal{P}$ is a convex polyhedron, $\operatorname{Terr}(\mathcal{F})$ is a convex polyhedron bounded by $\mathcal{F}$ and the medial axis $\mathcal{M}$ of $\mathcal{P}$. Let $J$ be a point of the medial axis closest to $X$. Observe that $J$ cannot be on an edge of the medial axis since $\operatorname{Terr}(\mathcal{F})$ is convex. Let $\mathcal{H}$ be the support plane of the facet of the medial axis containing $J$. The maximal ball centered at $J$ touches $\mathcal{S}$ in two facets $\mathcal{F}$ and $\mathcal{F}^{\prime}$. We denote the angle between $\mathcal{F}$ and $\mathcal{F}^{\prime}$ by $\alpha \in[0, \pi$ [. In addition, we call $I$ the center of the maximal ball passing through $X$, and $K$ the intersection point of the plane $\mathcal{H}$ with the straight-line $(X I)$. Since $\operatorname{Terr}(\mathcal{F})$ is convex, $\|X I\| \leq\|X K\|$ and

$$
\frac{R}{\operatorname{lfs}(X)}=\frac{\|X I\|}{\|X J\|} \leq \frac{\|X K\|}{\|X J\|}=\frac{1}{\cos (\alpha / 2)} \leq \frac{1}{\cos \left(\alpha_{\max } / 2\right)}
$$




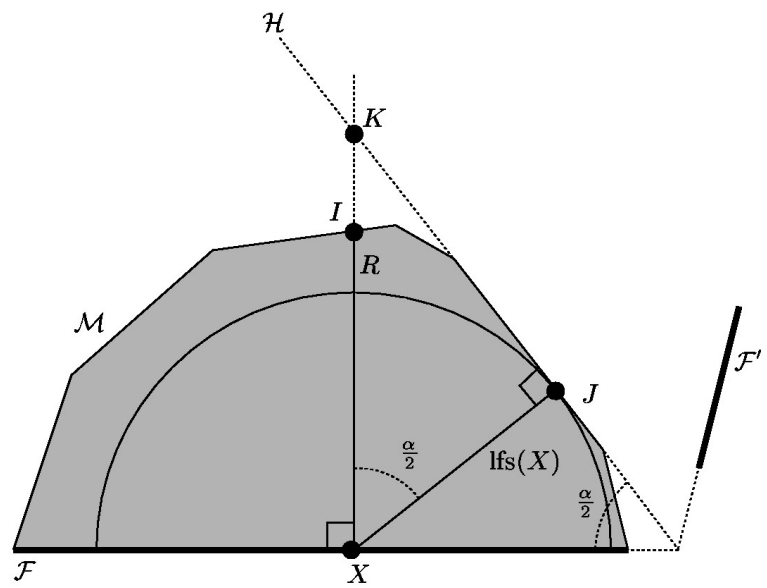

Fig. 5. For the proof of Property 13. The gray region represents the set of points nearest to $\mathcal{F}$ than to any other facet.

\section{2. $\quad$ Counting Delaunay Edges}

In what follows, $\mathcal{S}$ designates a polyhedral surface and $\mathcal{A}$ a light uniform $\varepsilon$-sample of $\mathcal{S}$. We count the Delaunay edges incident to $X \in \mathcal{A}$. We enclose $\mathcal{S}$ in a sufficiently large bounding box $\mathcal{B}$. Therefore, the radius of any maximal ball remains bounded. We denote the radius of the greatest maximal ball by $R_{\max }$. Moreover, we add points on $\mathcal{B}$ so that the union of these additional points and the sample points on $\mathcal{S}$ constitute a light uniform $\varepsilon$-sample of $\mathcal{B} \cup \mathcal{S}$. Observe that the total number of points remains $O(n)$.

We consider two different types of zones on the surface (see Fig. 6(a)), an $l$-singular zone surrounding singular points and an $l$-regular zone containing exclusively regular points.

Definition 14 ( $l$-Regular and $l$-Singular Zones). Let $l \geq 0$. We call the set of points $X \in \mathcal{S}$ such that lfs $(X)>l$ the $l$-regular zone of $\mathcal{S}$. We call the set of points that do not belong to the $l$-regular zone the $l$-singular zone of $\mathcal{S}$.

The 0 -singular zone (i.e., the set of singular points) of a polyhedral surface consists of its edges. For $l \neq 0$, the $l$-singular zone surrounds the edges of the polyhedral surface.

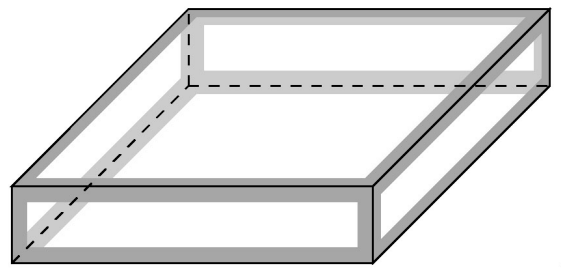

(a)

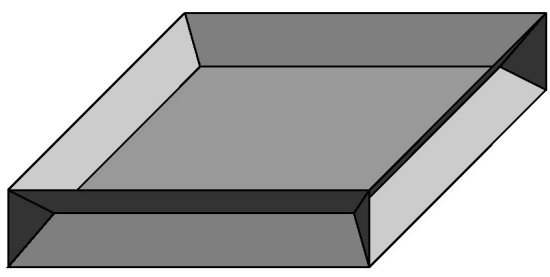

(b)

Fig. 6. (a) A polyhedral surface and its $l$-singular zone and (b) its medial axis. 
The width of the $l$-singular zone around an edge depends on the dihedral angle at this edge. The parameter $l$ will be fixed later.

In the $l$-regular zone, every point $X$ has only two types of Delaunay neighbors: neighbors that are "close" to $X$ and neighbors that are "close" to the two maximal balls through $X$ :

Proposition 15. Let $l^{3} \gg 4 R_{\max }^{2} \varepsilon$. Let $X$ be a sample point in the l-regular zone of $\mathcal{S}$ and $A$, a Delaunay neighbor of $X$. Let $B\left(I_{0}, R_{0}\right)$ and $B\left(I_{1}, R_{1}\right)$ be the two maximal balls through $X$. Then there exists $h_{i} \approx 20 R_{i}^{3}\left(\varepsilon / l^{3}\right)$ for $i \in\{0,1\}$ such that

$$
A \in B(X, \operatorname{lfs}(X)) \cup B\left(I_{0}, R_{0}+h_{0}\right) \cup B\left(I_{1}, R_{1}+h_{1}\right) .
$$

Proof. Let $V$ be any vertex of the Voronoi facet dual to the Delaunay edge [XA]. Let $I$ and $R$ be the center and the radius of the maximal ball through $X$ such that $\overrightarrow{X V} \cdot \overrightarrow{X I}>0$. We assume $\|X A\| \geq \operatorname{lfs}(X)$ and prove that there exists $h \approx 20 R^{3}\left(\varepsilon / l^{3}\right)$ such that $A \in B(I, R+h)$.

In order to apply Theorem 10, we have to check that $\|X Y\|^{2} / 4 R^{2} \gg \varepsilon /$ lfs $(X)$ and $\|X A\|^{2} / 4 R^{2} \gg \varepsilon / \operatorname{lfs}(X)$. However, by Property $12,\|X Y\| \geq \operatorname{lfs}(X)$ and

$$
\frac{\|X Y\|^{2}}{4 R^{2}} \geq \frac{\operatorname{lfs}(X)^{2}}{4 R^{2}} \geq \frac{l^{2}}{4 R_{\max }^{2}} \gg \frac{\varepsilon}{l} \geq \frac{\varepsilon}{\operatorname{lfs}(X)} .
$$

In a similar way,

$$
\frac{\|X A\|^{2}}{4 R^{2}} \geq \frac{\operatorname{lfs}(X)^{2}}{4 R^{2}} \geq \frac{l^{2}}{4 R_{\max }^{2}} \gg \frac{\varepsilon}{l} \geq \frac{\varepsilon}{\operatorname{lfs}(X)} .
$$

Therefore, and using the inequality $1 \leq R / \operatorname{lfs}(X)$,

$$
\|V I\| \lesssim R \frac{\varepsilon}{\operatorname{lfs}(X)} \max \left(\frac{10 R^{2}}{\operatorname{lfs}(X)^{2}},\left(1+\frac{2 R}{\operatorname{lfs}(X)}\right)^{2}\right) \lesssim 10 R^{3} \frac{\varepsilon}{l^{3}} .
$$

An upper bound on $\|I A\|$ follows immediately since, by the triangle inequality,

$$
\begin{aligned}
\|I A\| & \leq\|I V\|+\|V A\|=\|I V\|+\|V X\| \\
& \leq 2\|I V\|+\|I X\|, \\
\|I A\|-R & \lesssim 20 R^{3} \frac{\varepsilon}{l^{3}}
\end{aligned}
$$

Lemma 16. Letl $>2 \varepsilon$. Let $X$ be a point in thel-regular zone. The number of Delaunay neighbors of $X$ in $B(X, \operatorname{lfs}(X))$ is $O(1)$.

Proof. By Property 11, we know that $B(X, \operatorname{lfs}(X)) \cap \mathcal{S}$ is a disk $\mathcal{D}$. Let $A \in \mathcal{D}$ be a Delaunay neighbor of $X$. Let $\mathcal{B}$ be an empty ball passing through $X$ and $A$, i.e., a ball whose interior contains no sample points. $\mathcal{B}$ intersects the support plane of $\mathcal{D}$ in a disk centered at $C$. Let $I$ be the point of the half-line starting at $X$ and passing 
through $C$ such that $\|X I\|=\min (\|X C\|$, lfs $(X) / 2)$. By definition of $I, B(I,\|X I\|) \subseteq$ $B(X, \operatorname{lfs}(X))$. Because $B(X, \operatorname{lfs}(X))$ intersects the surface in a disk, $B(I,\|X I\|)$ also intersects the surface in a disk, and, since $B(I,\|X I\|) \subset \mathcal{B}, B(I,\|X I\|)$ is empty. Our sampling condition imposes that $\|X I\|=\min (\|X C\|$, lfs $(X) / 2) \leq \varepsilon$, which implies that $\|X C\| \leq \varepsilon$ since lfs $(X)>2 \varepsilon$. Therefore, $\|X A\| \leq 2\|X C\| \leq 2 \varepsilon$. Since the sample is light, the number of sample points at distance $2 \varepsilon$ from $X$ is $O(1)$.

Proposition 17 (Counting Edges in the $l$-Regular Zone). Let $l^{3} \gg 4 R_{\max }^{2} \varepsilon$. The number of Delaunay edges incident to a given sample point of the regular zone is $O\left(1 / \varepsilon l^{3}\right)$. The total number of edges incident to the l-regular zone is $O\left(1 / \varepsilon^{3} l^{3}\right)$.

Proof. Let $X$ be any point in the $l$-regular zone. Let $B\left(I_{0}, R_{0}\right)$ and $B\left(I_{1}, R_{1}\right)$ be the two maximal balls through $X$. By Proposition 15 , there exists $h_{i} \approx 20 R_{i}^{3}\left(\varepsilon / l^{3}\right)$ for $i \in\{0,1\}$ such that the Delaunay neighbors of $X$ belong to

$$
B(X, \operatorname{lfs}(X)) \cup B\left(I_{0}, R_{0}+h_{0}\right) \cup B\left(I_{1}, R_{1}+h_{1}\right) .
$$

By Lemma 16, the number of Delaunay neighbors in $B(X, \operatorname{lfs}(X))$ is $O(1)$. Let us prove that the number of Delaunay neighbors in $B\left(I_{i}, R_{i}+h_{i}\right)$ is $O\left(1 / \varepsilon l^{3}\right)$, for $i \in\{0,1\}$. Because the polyhedral surface has a bounded number of faces, the intersection of the enlarged maximal ball $B\left(I_{i}, R_{i}+h_{i}\right)$ with $\mathcal{S}$ is included in a bounded number of disks. Each disk has radius at most $r \approx \sqrt{2 R_{i} h_{i}} \approx R_{i}^{2} \sqrt{40 \varepsilon / l^{3}}$ (see Fig. 7). Therefore, the number of sample points in the enlarged maximal ball is $O\left(r^{2} / \varepsilon^{2}\right)=O\left(1 / \varepsilon l^{3}\right)$.

Proposition 18 (Counting Edges in the $l$-Singular Zone). Let $l>0$. The number of Delaunay edges joining two sample points in the $l$-singular zone is $O\left(l^{2} / \varepsilon^{4}\right)$.

Proof. Let $p$ be the length of the 0 -singular zone. The $l$-singular zone can be covered by $O(p / l)$ spheres of radius $2 l$. Therefore, the number of points in the $l$-singular zone is $O\left(p / l \times 4 l^{2} / \varepsilon^{2}\right)=O\left(l / \varepsilon^{2}\right)$. The number of edges joining two points of the $l$-singular zone is therefore $O\left(l^{2} / \varepsilon^{4}\right)$.

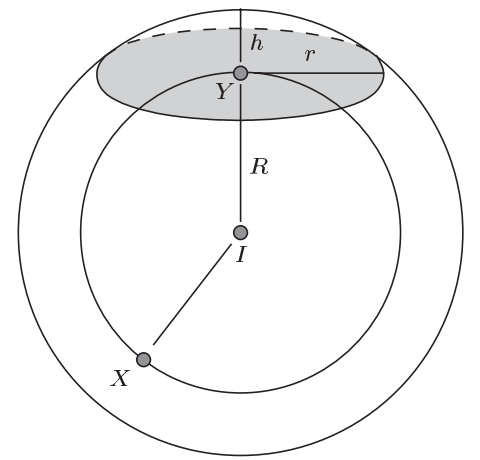

Fig. 7. A plane tangent to the ball $B(I, R)$ at $Y$ intersects $B(I, R+h)$ in a disk of radius $r=\sqrt{2 h R-h^{2}} \approx$ $\sqrt{2 R h}$ when $h \ll R$. 
In order to counterbalance the number of edges in the two zones, we have to choose $l$ such that $l^{2} / \varepsilon^{4}=1 / \varepsilon^{3} l^{3}$, in other words $l=\sqrt[5]{\varepsilon}$. We sum up our results in the following theorem:

Theorem 19. Let $\mathcal{A}$ be a light uniform $\varepsilon$-sample of a bounded polyhedral surface $\mathcal{S}$ of $\mathbb{R}^{3}$. The number of tetrahedra of the Delaunay triangulation of $\mathcal{A}$ is $O\left(n^{9 / 5}\right)=O\left(n^{1.8}\right)$.

\section{Convex Polyhedral Surfaces}

For convex polyhedral surfaces, we know from Property 13 that the ratio $R / \operatorname{lfs}(X)$ is bounded from above by a certain constant $C=\left(\cos \left(\alpha_{\max } / 2\right)\right)^{-1}$ where $\alpha_{\max }<\pi$ represents the greatest angle between any two facets. Using the same scheme as before, one can prove that the complexity of convex polyhedral surfaces is $O(n \sqrt{n})$. Again, we consider two different zones on the convex polyhedral surface.

Proposition 20. Let $l \gg 4 C^{2} \varepsilon$. Let $X$ be a sample point in the l-regular zone of $\mathcal{S}$ and $A$, a Delaunay neighbor of $X$. Let $B\left(I_{0}, R_{0}\right)$ and $B\left(I_{1}, R_{1}\right)$ be the two maximal balls through $X$. Then there exists $h \approx 20 C^{2} \varepsilon$ such that

$$
A \in B(X, \operatorname{lfs}(X)) \cup B\left(I_{0}, R_{0}+h\right) \cup B\left(I_{1}, R_{1}+h\right) .
$$

Proof. Let $V$ be any vertex of the Voronoi facet dual to the Delaunay edge [XA]. Let $I$ and $R$ be the center and the radius of the maximal ball through $X$ such that $\overrightarrow{X V} \cdot \overrightarrow{X I}>0$ and assume $\|X A\| \geq \operatorname{lfs}(X)$. Let us prove that there exists $h \approx 20 C^{2} \varepsilon$ such that $A \in B(I, R+h)$.

Using $1 / 4 C^{2} \gg \varepsilon / l$ and Property 12 , we get

$$
\frac{\|X Y\|^{2}}{4 R^{2}} \geq \frac{\operatorname{lfs}(X)^{2}}{4 R^{2}} \geq \frac{1}{4 C^{2}} \gg \frac{\varepsilon}{l} \geq \frac{\varepsilon}{\operatorname{lfs}(X)} .
$$

In a similar way,

$$
\frac{\|X A\|^{2}}{4 R^{2}} \geq \frac{\operatorname{lfs}(X)^{2}}{4 R^{2}} \geq \frac{1}{4 C^{2}} \gg \frac{\varepsilon}{l} \geq \frac{\varepsilon}{\operatorname{lfs}(X)} .
$$

Therefore, by Theorem 10 and using $1 \leq C$,

$$
\begin{aligned}
\|V I\| & \lesssim R \frac{\varepsilon}{\operatorname{lfs}(X)} \max \left(\frac{10 R^{2}}{\operatorname{lfs}(X)^{2}},\left(1+\frac{2 R}{\operatorname{lfs}(X)}\right)^{2}\right) \\
& \lesssim \max \left(10 C^{2},(1+2 C)^{2}\right) \varepsilon \\
& \lesssim 10 C^{2} \varepsilon
\end{aligned}
$$

and

$$
\|I A\|-R \lesssim 20 C^{2} \varepsilon
$$


Proposition 21 (Counting Edges in the $l$-Regular Zone). Let $l \gg 4 C^{2} \varepsilon$. Let $\mathcal{A}$ be a light uniform $\varepsilon$-sample. The number of Delaunay edges incident to a given sample point of the l-regular zone is $O(\sqrt{n})$. The total number of edges incident to the l-regular zone is $O(n \sqrt{n})$.

Proof. By Lemma 16, the number of Delaunay neighbors in $B(X, \operatorname{lfs}(X))$ is $O(1)$. Let us prove that the number of points in the enlarged maximal ball $B(I, R+h)$ is $O(\sqrt{n})$ where $h \approx 20 C^{2} \varepsilon$. The intersection of the enlarged maximal ball with $\mathcal{S}$ is included in a bounded number of disks. Each disk has radius at most $r \approx \sqrt{2 R h}$ (see Fig. 7). Therefore, the number of sample points in the enlarged maximal ball is $O\left(r^{2} / \varepsilon^{2}\right)=O(1 / \varepsilon)=O(\sqrt{n})$.

The counting of edges in the $l$-singular zone is unchanged and is given by Proposition 18. In order to find $O(n \sqrt{n})=O\left(1 / \varepsilon^{3}\right)$ edges in the singular zone, we have to choose $l^{2} / \varepsilon^{4}=1 / \varepsilon^{3}$, in other words, $l=\sqrt{\varepsilon}$.

Theorem 22. Let $\mathcal{A}$ be a light uniform $\varepsilon$-sample of a bounded convex polyhedral surface $\mathcal{S}$ of $\mathbb{R}^{3}$. The number of tetrahedra of the Delaunay triangulation of $\mathcal{A}$ is $O(n \sqrt{n})$.

\section{Conclusion}

We have given a subquadratic bound on the complexity of the Delaunay triangulation of points distributed on polyhedral surfaces. Our proof is based on the fact that a subset of the Voronoi vertices of a sample are close to the medial axis of the surface. This result of independent interest also holds for smooth surfaces. An obvious open question is to extend our results to smooth surfaces. Bounding the number of tetrahedra in the case of smooth surfaces seems to be harder, due to the presence of slivers, i.e. flat tetrahedra whose circumcenters can be arbitrarily far from the medial axis. Observe that Theorem 10 indicates that these tetrahedra are small.

Note. Since the publication of this paper, we established a linear bound for polyhedral surfaces using a different approach. ${ }^{1}$ Golin and $\mathrm{Na}$ also established a new result in the probalistic context. $^{2}$

\section{References}

1. N. Amenta and M. Bern. Surface reconstruction by voronoi filtering. Discrete Comput. Geom., 22:481-504, 1999.

${ }^{1}$ D. Attali and J.-D. Boissonnat. A linear bound on the complexity of the Delaunay triangulation of points on polyhedral surfaces. In Proc. 7th ACM Symposium on Solid Modeling and Applications, pages 139-145, 2002.

${ }^{2}$ M. J. Golin and Hyeon-Suk Na. The probabilistic complexity of the voronoi diagram of points on a polyhedron. In Proc. 14th Annual ACM Symposium on Computational Geometry, pages 209-216, 2002. 
2. N. Amenta and R. K. Kolluri. The medial axis of a union of balls. Comput. Geom. Theory Appl., 20:25-37, 2001.

3. A. G. Belyaev, E. V. Anoshkina, and T. L. Kunii. Ridges, ravines and singularities. In A. T. Fomenko and T. L. Kunii, editors, Topological Modeling for Visualization. Springer-Verlag, New York, 1997.

4. J.-D. Boissonnat and F. Cazals. Natural neighbor coordinates of points on a surface. Comput. Geom. Theory Appl., 19:155-173, 2001.

5. J.-D. Boissonnat and F. Cazals. Smooth surface reconstruction via natural neighbour interpolation of distance functions. Comput. Geom. Theory Appl., 21:185-203, 2002.

6. L. Calabi and J. A. Riley. The skeletons of stable planesets. Technical Report, Parke Math. Lab. Inc., One River Road, Carlisle, Massachusetts, December 1967.

7. T. M. Chan, J. Snoeyink, and C. K. Yap. Primal dividing and dual pruning: output-sensitive construction of four-dimensional polytopes and three-dimensional Voronoi diagrams. Discrete Comput. Geom., 18:433454, 1997.

8. R. A. Dwyer. Higher-dimensional voronoi diagram in linear expected time. Discrete Comput. Geom., 6:343-367, 1991.

9. R. A. Dwyer. The expected number of $k$-faces of a voronoi diagram. Internat. J. Comput. Math., 26(5):1321, 1993.

10. J. Erickson. Nice point sets can have nasty delaunay triangulations. In Proc. 17th Annual ACM Symposium on Computational Geometry, pages 96-105, 2001.

11. M. Golin and Hyeon-Suk Na. On the average complexity of 3d-voronoi diagrams of random points on convex polytopes. In Proc. 12th Canadian Conference on Computational Geometry, pages 127-135, 2000.

12. Hyeong In Choi, Sung Woo Choi, and Hwan Pyo Moon. Mathematical theory of medial axis transform. Pacific J. Math., 181(1):57-88, 1997.

13. G. Matheron. On the negligibility of the skeleton and the absolute continuity of erosions. In J. Serra, editor, Image Analysis and Mathematical Morphology, volume 2. Academic Press, London, 1988.

14. J. Riley and L. Calabi. Certain properties of circles inscribed in simple closed curves. Technical Report 59281, Parke Math. Lab. Inc., One River Road, Carlisle, Massachusetts, 1964.

Received July 27, 2001, and in revised form September 24, 2002. Online publication July 25, 2003. 